\title{
An Artificially Intelligent Algorithmic Paradigm for Risk Mitigation in Risk Man- aged Software Testing
}

\author{
Vinita Malik \\ Central University of Haryana \\ India \\ is@cuh.ac.in \\ Sukhdip Singh \\ D. C. R. U. S. T, Murthal \\ India \\ sukhdeepsingh.cse@dcrustm.org
}

\begin{abstract}
Various iterations of testing of the software are required for fulfilling specific quality parameters which further helps in quality management of any software. The heart of this research pumps for a Neuro Fuzzy Framework that will be utilized for mitigating software risks and such risk mitigation will lead to test case prioritization. This approach for risk mitigation has been considered due to high accuracy levels. Here the system will be trained by Bayesian regulation approach due to high prediction capabilities. Requirements identification has been done for the development of prototype of the tool which will determine risk levels. Once requirements are identified then by risk analysis the testing effort will be estimated properly for software quality improvement. The algorithmic paradigm has been developed for the proposed system which will be implemented by MATLAB or by Java Frameworks. Fuzzy logic has been employed to make fuzzy inference system.
\end{abstract}

Keywords: Fuzzy Logic, Back Propagation Technique, Risk Based Testing, Risk Management, Bayesian Regulation Technique

Received: 19 May 2018, Revised 1 July 2018, Accepted 7 July 2018

DOI: $10.6025 / \mathrm{jdp} / 2018 / 8 / 3 / 100-105$

(C) 2018 DLINE. All Rights Reserved

\section{Introduction}

Software quality management is a continuous process for establishing a type of environment which allows top management officials for doing evolutionary testing of the software [1]. The software is released after code inspection or review is done. The software testing is done properly for detecting as well as fixing all the issues before getting any kind of failures [2]. Simplest techniques for the failure identification include past experience or brainstorming. For removal of failures in software development life cycle all the project risks must be identified as early as possible. Risk has been characterized by two factors by checking the potential failure severity level and by finding probability of occurring the event [3]. The consequence is thought as output of forward risks and probability of occurrence as an output of backward risks [4]. Risks are categorized as requirements based, project management based, business, quality, relational, decision making based, technical issues based .All of these risks are derived by software availability, security, reliability, safety, resilience[5].

\begin{tabular}{llllll}
\hline 100 & Journal of Data Processing & Volume 8 & Number 3 & September 2018 \\
\hline
\end{tabular}


In the present research, an idea about a hybrid Neuro fuzzy technique is given for risk assessment which will further help in test prioritization. In the first stage or in the learning phase of the system will act like a neural net and will be able to learn its parameters in the offline mode and in the second stage it will act as a fuzzy system. Neural nets will help in improving the accuracy of the system and fuzzy system will make all the computations flexible. As the process environment is evolutionary in nature so consistent adjustment of software tool is required. Various search engines have been employed for getting our results i.e. Springer, Elsevier, IEEE, Google Scholar. The research has been divided into various sections. In section 1, research aims have been introduced and in the section 2, focus on some of risk management techniques and risk based testing has been provided. In next section some of the artificial intelligence techniques have been explained used in past for risk mitigation. In section 4, proposed algorithm has been discussed and finally conclusion is provided in the last section with explanation of future scope.

\section{Software Risk Management and Risk Managed Testing}

\subsection{Risk Management}

Risk management is comprised of following 4 stages [6]:

- Risk Identification: For identification of all risk factors of projects.

- Risk Analysis: For analyzing and assessing risk involved in the software projects.

- Risk Planning: For drawing out plan how to mitigate risk.

- Risk Monitoring: By employing various risk reduction methodologies.

\subsection{Various Methodologies for Risk Management}

Some of the general methodologies that are used for risk management have been discussed as follows [7]:

- Checklist: For making a list of top risk factors and a low cost solution.

- Analytical Frameworks: Various target areas are defined which may be used to reduce various kinds of risks as per technology, expertise and team communication.

- Process Models: Various models are used for proper sequencing of risk management activities.

\subsection{Risk Managed Testing}

Risk managed testing may be composed of various stages by which risk is managed well in the software projects. The generic risk managed/oriented testing is seen in the figure 1 [8] as follows:

\section{Literature Survey}

In the project management the Risk control has been amongst one of the most important issue .Risk analysis is needed after risk identification for analyzing all risk factors, once analysis is done further optimization is done. Particle swarm optimization technique has been used in the past for risk analysis in the past [9].

New products are screened by a 3-tuple fuzzy approach as they are used to find out all incomplete and vague information and for tackling vague and complex system. So linguistic assessment is to done by fuzzy linguistic approach. Such method is employed for new products and the results are achieved by unifying the important weights and the preferences of average in nature. The average extent of ignoring information of the given criteria is utilized here [10].

Risk Mitigation and Risk planning are considered for resolving all issues related to risk items. As Risk mitigation is really crucial for most of the software projects and is also our primary concern. Fuzzy logic is used to utilize the results from incomplete information. For determination of degree of truth membership functions are to be given due importance.

An early warning system has been employed by using fuzzy logic approach. Software projects get failed if all the risks in the projects are identified very late in the system. So for managing risks early in SDLC an early warning system is utilized which is based on fuzzy logic software metrics. It has helped in resolution of all conflicts in an uncertain environment while doing assessment of the risks [11]. 


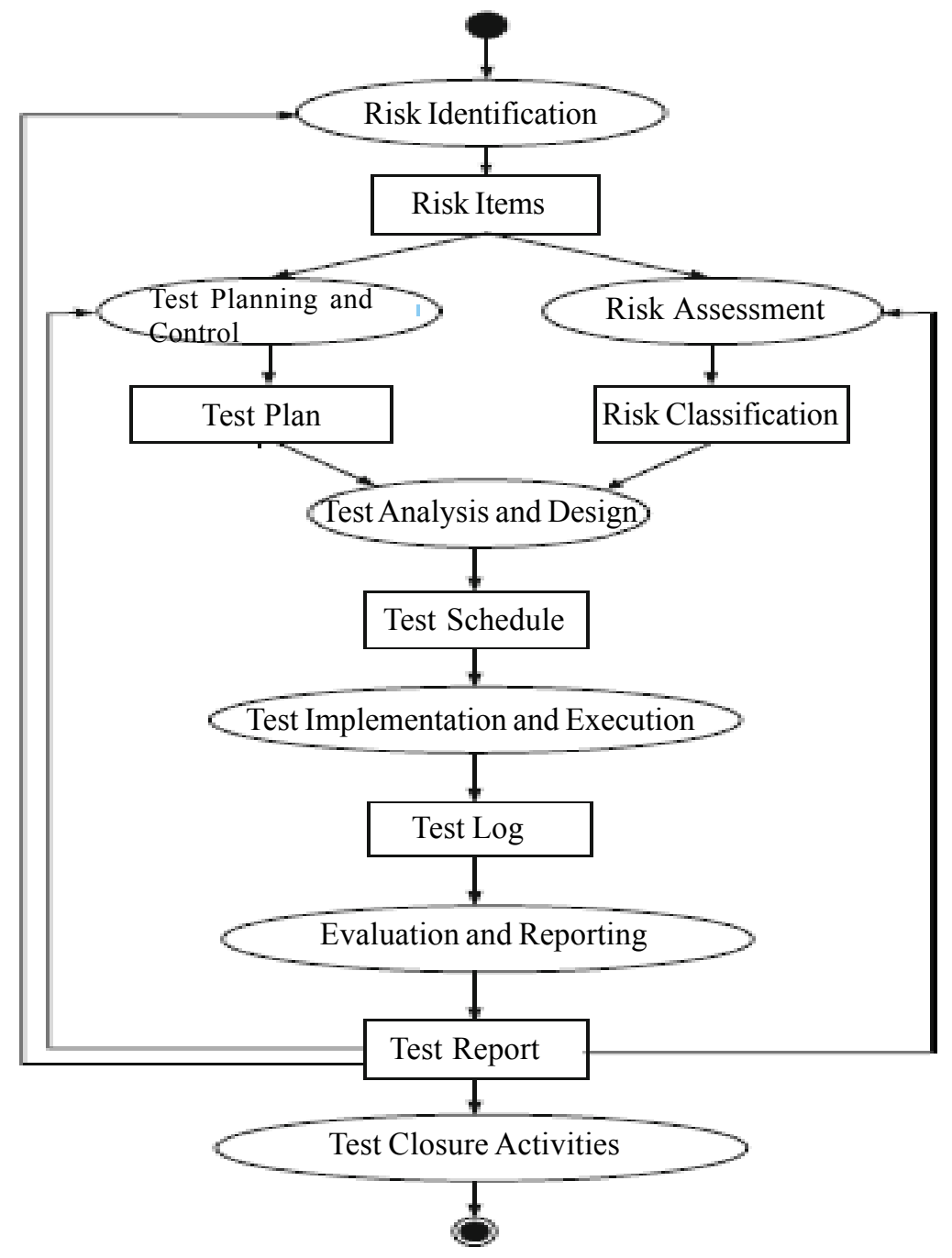

Figure 1. A quite generic process of risk oriented testing [8]

Impact Ratings are utilized as an effective tool for fuzzy risks. The risk exposure level is to be considered for a very new system development project. FLRAM process top design Evaluation Framework has been considered in the past [12].

Neural networks are also employed for dynamic information processing. All interconnected brain cells are activated for recognizing patterns and for decision making. The interconnected processing of elements happens by their state response to external inputs in dynamic manner [13].

Genetic programming has been used in past for reduction of risks in the project [14]. Decision trees have been used in combination with genetic programming for delivering the software project in allotted resources.

Modified Expected Cost Misclassification is to be minimized and need to optimize fault prone modules. The risk estimation accuracy is improved if Bayesian network approach based on PSO is used [15]. Effort estimation for various types of software has been done by various modeling techniques and which have been further used for fitting the unclear properties of software [16]. Software effort is estimated if the artificial neural nets are embedded into Fuzzy Inference system. The Neuro-fuzzy systems have been categorized into various categories like student modeling system, economic system, electrical and electronics system. The ways that exists for developing ANN are waterfall model or spiral model which focuses on each phase of software development. Adaptive neuro fuzzy models have categorized work rate on basis of 4 categories i.e. very light, light, moderate, heavy [17].

\begin{tabular}{lllll}
\hline 102 & Journal of Data Processing & Volume 8 & Number 3 & September 2018 \\
\hline
\end{tabular}


Neuro Fuzzy classifiers have been used in the past to for lowering down the complexity of internet flows [18] .It has used privacy thread as well as usage and Neuro Fuzzy learning in case of Min-Max networking which is trained by usage of Parc algorithm. Such technique was used for building the quality of service more valuable.

The grain moisture charges have been described by Neuro fuzzy model in image capturing device [19, 20]. This Model used Back propagation Algorithm for training purpose. The human process operator helped in building the If then Rules which were utilized as the membership function. This system used Adaptive network for fuzzification process. Neuro fuzzy methodologies have been used in the past for tackling with several business risks [21]. Various cost risks have been identified and mitigated with the help of COCOMO II model and neuro fuzzy techniques [22]. Management related risks have been identified and assessed in past using the fuzzy inference 3-D model which has proved a really efficient approach [23]. Cuckoo algorithm with Fuzzy sets has been used to find out uncertainty levels in case of software cost estimation which is a non-algorithmic paradigm [24].

Big data risks related to data and speed are also minimized with the help of neuro fuzzy framework to accommodate in an intelligent system known as ANFIS [25].

\section{Proposed Algorithm}

In this section we are proposing an algorithm based on neuro fuzzy system for risk mitigation. Firstly a fuzzy inference system is built. Following steps are required for creating the system:

- Build all rules encompassing if then statements which are to be utilized for doing Fuzzification.

- All the rules will be built up by MATLAB /Java Frameworks.

- All the project risk items are considered which include evolving requirements, poor plan, and schedule issues.

-Apply fuzzification on input risk items by variables i.e. Low/Medium/High for all the inputs.

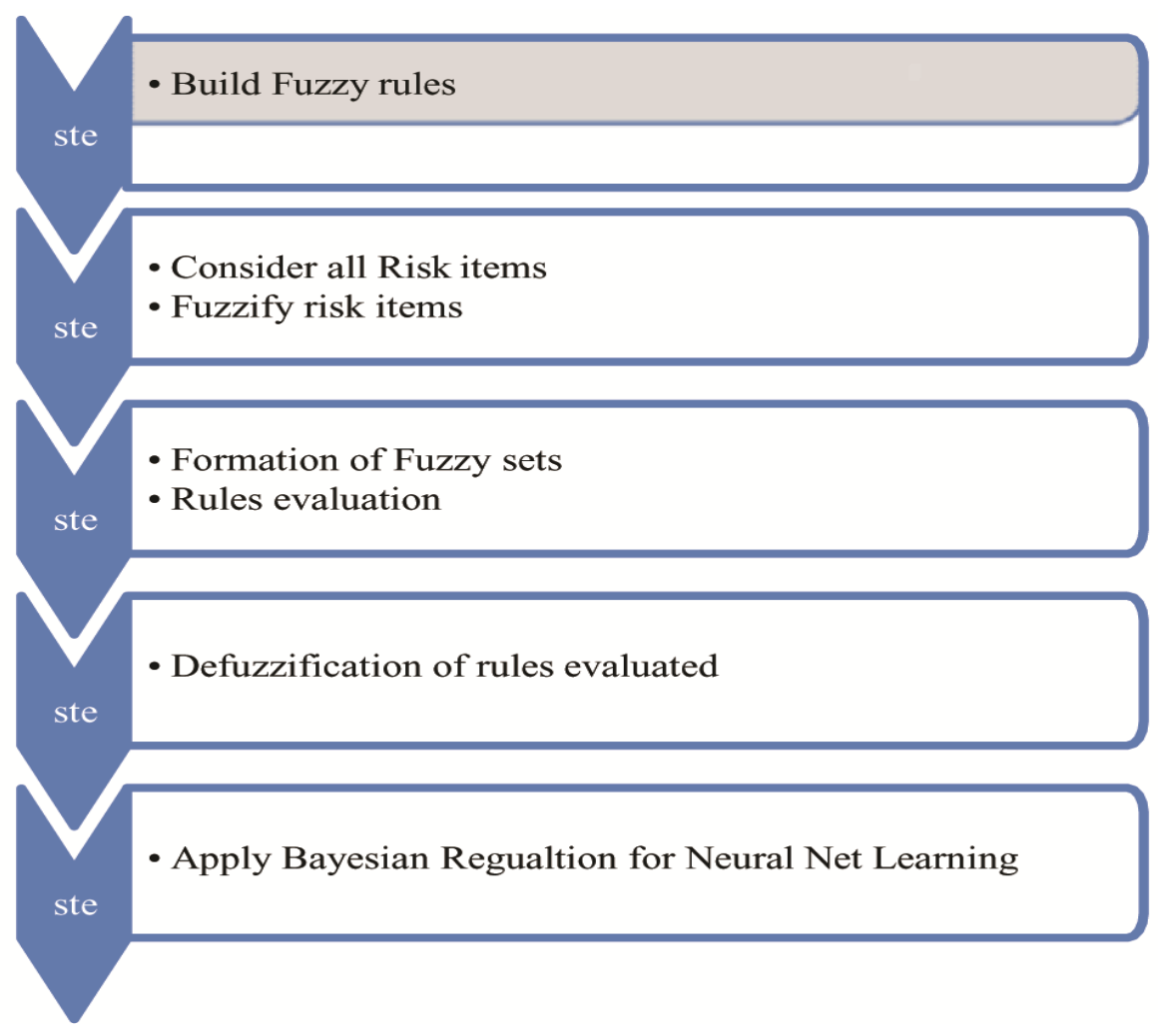

Figure 2. Proposed Algorithm 
- All variables are required to be represented in the form of sets which may be named as fuzzy sets and are considered for evaluation.

- Sets are created under the consideration of membership functions.

- Now the rules are required to be evaluated.

- Apply defuzzification for evaluation of the rules output.

As the fuzzy system has been established so now neural networks are used for giving provision of learning ability to the system. Bayesian Regulation technique is applied over Back propagation algorithm due to greater prediction capabilities. The complete algorithmic process is shown below in five steps process by figure 2 :

For example, Expert COCOMO model cost drivers are considered and the results may be derived based on the proposed algorithm in future.

\section{Conclusions and Future Work}

Risks in the projects require early detection of faults for safe system development and minimal project losses. Various artificial intelligent techniques have been explored for mitigating risk of the project but it has been found that Neuro Fuzzy paradigm will be the best approach for dealing with all risk items of the project as it will lead to more accurate results and high prediction capabilities for the system under consideration. After creation of fuzzy inference model, Bayesian regulation technique is used for better system performance. An algorithmic paradigm has been proposed based on Neuro Fuzzy technique. In future, this proposed methodology may be implemented via MATLAB/Java Frameworks and can be applied on various software projects for risk mitigation.

\section{References}

[1] Mathrani, A. (2014). Quality Assurance Strategy for distributed software development using Managed test lab model, IEEE.

[2] Hemmati, H., Nagappan, M. (2014). Investigating the effect of defect co fix on quality assurance resource allocation: $A$ search based Approach, Elsevier.

[3] Rosenberg, L., Stapko, R., Gallo, A. (1999). Risk-based object oriented Testing, Proceeding of the 24th Annual Software Engineering Workshop, p. 1-6. NASA, Software Engineering Laboratory.

[4] Redmill, F. (2004). Exploring risk-based testing and its implications, Software Testing, Verification and Reliability. V. 14, 3-15

[5] Felderer, M., Schieferdecker, I. (2014). A taxonomy of risk-based testing, International Journal on Software Tools for Technology Transfer, 16. 559-568.

[6] Alam, M., Khan, A. (2013). Risk-based testing techniques: a perspective Study, International Journal of Computer Applications. 65, 33-41.

[7] Bannerman, P. L. (2008). Risk and risk management in software projects: A reassessment, The Journal of Systems and Software, 2(2) 442-450.

[8] Felderer, M., Ramler, R. (2014). Integrating risk-based testing in industrial test processes, Springer Science+ Business Media, Software Quality Journal, 22. 543-575

[9] Cao, P., Chen, F. (2009). A Risk Control Optimization Model for Software Project, IEEE.

[10] Guo, W., Huynh, G., Nakamori, Y. (2016). A Proportional, 3- Tuple Fuzzy Linguistic Representation Model, Springer.

[11] Liu, X., Kane, G., Bambroo, M. (2003). An Intelligent Early Warning System for Software Quality Improvement and Project Management, IEEE Computer Society.

[12] Hsieh, M., Hsu, Y., Lin, C. (2016). Risk assessment in new software development projects at the front end: a fuzzy logic approach, Springer.

[13] Rodvold, M. (1999). A Software Development Process Model for Artificial Neural Networks in Critical Applications, IEEE.

\begin{tabular}{lllll}
\hline 104 & Journal of Data Processing & Volume 8 & Number 3 & September 2018 \\
\hline
\end{tabular}


[14] Khoshgoftaar, T. (2007). A Multi-Objective Software Quality Classification Model Using Genetic Programming, IEEE Transactions.

[15] Sandhu, G., Salaria, D. A. (2014). Bayesian Network Model of the Particle Swarm Optimization for Software Effort Estimation, International Journal of Computer Applications.

[16] Huang, S., Chiu, N. (2007). Applying fuzzy neural network to estimate software development effort, Springer.

[17] Kolus, A., Imbeau, D., Dube, P., Dubeau, D. (2016). Classifying work rate from heart rate measurements using an adaptive neuro-fuzzy inference system, Elsevier.

[18] Rizzi, A., Iacovazzi, A. (2015). A low complexity real-time Internet traffic flows neuro-fuzzy classifier, Elsevier.

[19] Virgen-Navarroa, L. (2016). Neuro-fuzzy model based on digital images for the monitoring of coffee bean color during roasting in a spouted Bed, Science Direct.

[20] Malik. V., Manchanda. G. (2017). An algorithmic design to mitigate risks by neuro fuzzy techniques, IJARCS, 8, p. $22-27$

[21] Rajab, S., Sharma, V., Artif Intell Rev. (2017). A review on the applications of neuro-fuzzy systems in business, Springer Science + Business Media Dordrech.

[22] Kaur, I., Narula, G. S., Wason. (2018). Neuro fuzzy—COCOMO II model for software cost estimation, International Journal of Information Technology, 1-7.

[23] Hawas, Y. E., Al-Nahyan, M. T. (2017). A Fuzzy-Based Approach to Estimate Management Processes Risks. In: Meier A., Portmann E., Stoffel K., Terán L. (eds) The Application of Fuzzy Logic for Managerial Decision Making Processes. Fuzzy Management Methods. Springer, Cham, 73-84.

[24] Kaushik, A., Verma, S., Singh, H. J. (2017). International Journal of System Assurance Engineering and Management, 8 (2) 1461-1471.

[25] Taneja, R., Gaur, D. (2018). Robust Fuzzy Neuro system for Big Data Analytics. In: Aggarwal V., Bhatnagar V., Mishra D. (eds) Big Data Analytics. Advances in Intelligent Systems and Computing, 654. Springer, Singapore. 